\title{
INFLUENCE OF GRAVITATION ON HEAT TRANSFER BY NATURAL CONVECTION
}

\author{
Arnout Willockx ${ }^{1}$, Gilbert De Mey ${ }^{2}$, Michel De Paepe ${ }^{1}$, Boguslaw Wiecek ${ }^{3}$, Mariusz Felczak ${ }^{3}$, Mario De \\ Craemer $^{4}$, Arnaud Stouff ${ }^{4}$, C. Van Rumst ${ }^{4}$ \\ ${ }^{1}$ Department Of Flow, Heat and Combustion Mechanics, Ghent University \\ Sint-Pietersnieuwstraat 41, 9000 Gent, Belgium \\ Arnout.Willockx@ugent.be \\ ${ }^{2}$ Department of Electronics and Information Systems, Ghent University \\ Sint-Pietersnieuwstraat 41, 9000 Gent, Belgium \\ ${ }^{3}$ Institute of Electronics, Technical University of Lodz \\ B. Stefanowskiego 18/22, 90-924 Lodz, Poland \\ ${ }^{4}$ Department of Industrial Sciences BME-CTL, Hogeschool Gent, \\ Schoonmeersstraat 52, 9000 Gent, Belgium
}

\begin{abstract}
The objective is to separate natural convection and radiation experimentally. Therefore a heat source is placed inside a closed cavity and the acceleration inside the cavity can be changed. A centrifuge is used to change the acceleration. A flat resistor etched on a printed circuit board of $25.5 \mathrm{~mm} \times$ $25.5 \mathrm{~mm}$, is placed in a hermetically sealed cylinder, which hangs under the arm of the centrifuge. The resistor is powered by batteries, dissipates $1.63 \mathrm{~W}$ and has a surface temperature of $82^{\circ} \mathrm{C}$ at $1 \mathrm{~g}$. Natural convection is maintained inside the cylinder. Convection and radiation are the main modes of heat transfer, but there's also an amount of conduction through the wires that connect the batteries with the resistor. The rotational speed of the centrifuge determines the centrifugal force in the cylinder. When the centrifugal force increases, the temperature of the resistor decreases due to the increase of natural convection. The amount of radiation, conduction and total heat transfer can be determined from the experiment, so the amount of natural convection can also be determined. The experimental results are compared with the governing equations to validate the experiment. The reproducibility of the experiment is also checked.
\end{abstract}

Keywords: Rotational forces, natural convection, radiation, experimental.

\section{Introduction}

There is little published research on this subject. Most studies look at the influence of gravity modulation on natural convection in closed cavities with no heat source in the cavity. A temperature difference is created between the walls of the cavity to obtain natural convection [1]. The temperature gradient can be lateral or vertical. Jin and Chen [2] used vertical slots with aspect ratio of 20 and 15 . They concluded that the gravity modulation had little effect on the heat transfer, but mostly affected the flow patterns in the slot. The flow pattern was the main subject in this paper. $\mathrm{Li}$ [3] investigated $\mathrm{g}$-jitter induced flows in microgravity and in a transverse magnetic field. This was done analytically and for a simple system of two plates.

Aside from gravity modulation there are also many studies about natural convection in vertical slots with constant acceleration (gravity). The gravity acceleration is either equal to $\mathrm{g}$ or much smaller than $\mathrm{g}$ (microgravity). This research has been done by numerical simulations [4] and experimental studies [5]. Other studies have been done in microgravity to measure fluid characteristics [6].

The method studied in this article has another objective. It is the intention to create a method to separate radiation from natural convection and conduction experimentally. A heat source is placed inside a cavity with homogenous wall temperature to induce natural convection. The acceleration inside the cavity can be changed from $1 \mathrm{~g}$ to $6 \mathrm{~g}$. There is no gravity modulation, but each measurement point has a different but constant value of acceleration. The surface temperature of the heat source, the ambient temperature in the cavity and the dissipated power in the heat source are measured at different accelerations. The radiative heat transfer from the heat source to the environment can be determined with these temperatures. The total heat transfer from heat source to environment is also known because it is equal to the power dissipated in the heat source. Conduction through the contact wires is also important. The difference between total heat transfer and radiation and conduction is the amount of natural convection. The experiment makes it possible to determine the amount of radiation and natural convection at different accelerations directly from the results, without using calculations or formulas. These results can be presented graphically and compared to the existing governing equations to validate the exactness of the experiment.

\section{Nomenclature}

$\begin{array}{ll}A_{\text {enc }} & \text { surface area of the container }\left[\mathrm{m}^{2}\right] \\ A_{w} & \begin{array}{l}\text { heat exchanging surface }=\text { surface area of the } \\ \text { resistor }\left[\mathrm{m}^{2}\right]\end{array} \\ A_{\text {wire }} & \begin{array}{l}\text { surface area of the profile of length of the connecting } \\ \text { wires }\left[\mathrm{m}^{2}\right]\end{array} \\ G r_{x} & \begin{array}{l}\text { Grashof number at height x } \\ H_{1}\end{array} \quad \begin{array}{l}\text { distance between resistor in cylinder and top of the } \\ \text { centrifuge }[\mathrm{m}]\end{array}\end{array}$

$A_{e n c} \quad$ surface area of the container $\left[\mathrm{m}^{2}\right]$ 


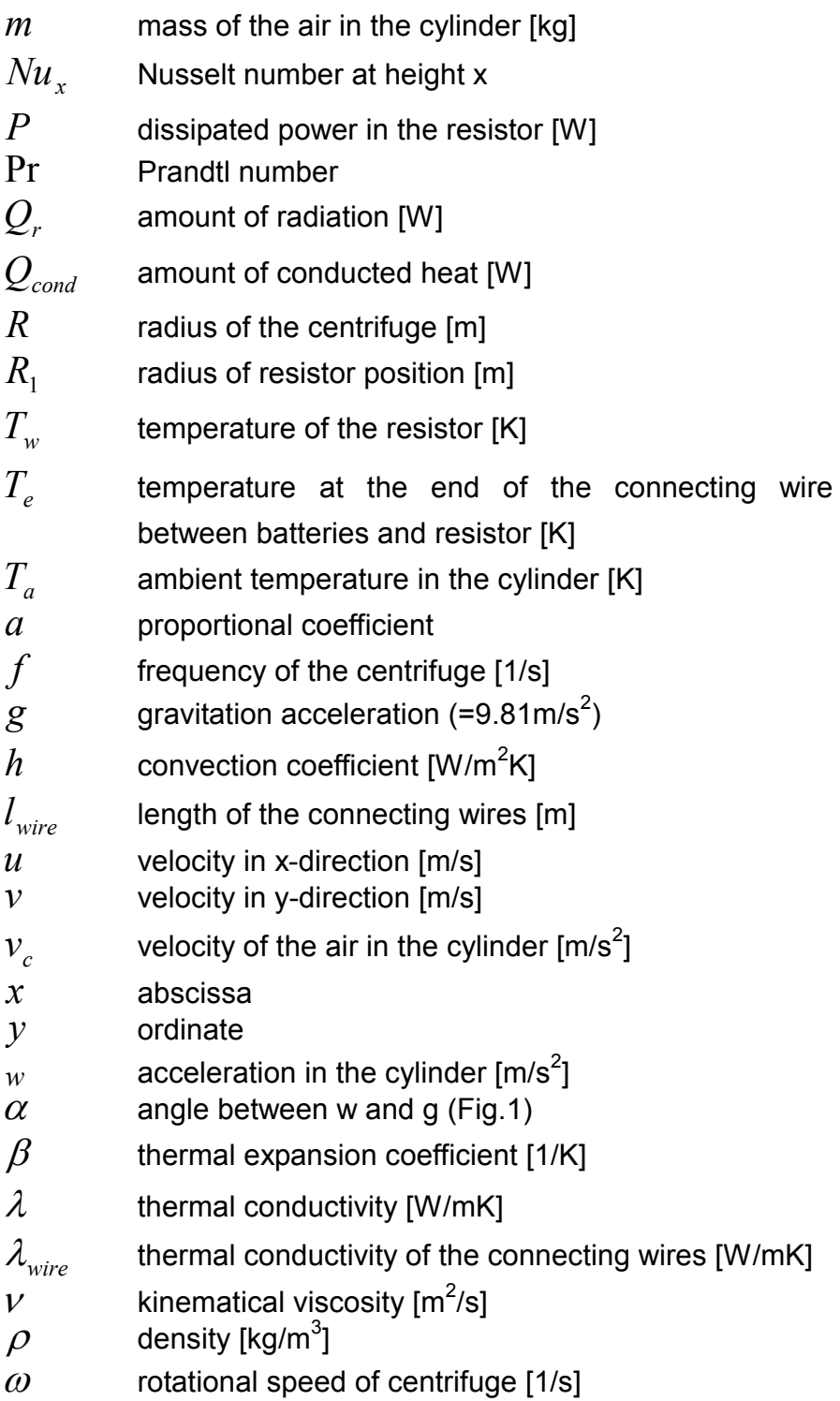

\section{Governing Equations}

To determine a physical description of the experiment, the boundary layer equations for free convection, with the Boussinesq approximation, are used. This means that all variable-property effects are neglected, except for density in the momentum equation. In the experiment, the heat source is a flat resistor. Therefore the equations for laminar flow on a constanttemperature, vertical, flat plate are used, with the $\mathrm{x}$-axis along the plate and the $y$-axis perpendicular on the plate. The equations are derived in [8]. The resulting Nusselt number is:

$$
N u_{x}=0.508\left(\frac{\operatorname{Pr}^{2}}{0.952+\operatorname{Pr}}\right)^{1 / 4} G r_{x}^{1 / 4}
$$

The Nusselt and Grashof number are replaced by their expressions:

$$
\begin{aligned}
& N u_{x}=\frac{h \cdot x}{\lambda} \\
& G r_{x}=\frac{g \cdot \beta \cdot\left(T_{w}-T_{a}\right) \cdot x^{3}}{v^{2}}
\end{aligned}
$$

Radiation from the resistor to the environment also has to be taken into account. The amount of radiation can be determined by:

$$
Q_{r}=2 \sigma A_{w}\left(T_{w}^{4}-T_{a}^{4}\right)
$$

where it is assumed that the emissivity of the resistor and the enclosure are both 1 . This assumption is approximate, but allows the amount of radiation to be estimated. It is an overestimation of the real radiation and this has to be kept in mind in the analysis of the experimental results. The radiating surface of the resistor is $2 A_{w}$, because the back of the resistor is free and has the same temperature as the front.

The dissipated power $(P)$ in the resistor is transferred to the environment by natural convection, conduction and radiation. Natural convection occurs on both sides of the resistor, so the heat transfer surface is $2 A_{w}$. A small part of the heat is transferred by conduction through the two small copper wires that connect the battery and resistor.

$$
Q_{\text {cond }}=\frac{2 \cdot A_{\text {wire }} \cdot \lambda_{\text {wire }} \cdot\left(T_{w}-T_{e}\right)}{l_{\text {wire }}}
$$

It is also important to notice that at $w=0$, there will be no air movement in the cylinder and heat will be transferred by conduction through the air. The dissipated power can be written as:

$$
P=h \cdot 2 A_{w}\left(T_{w}-T_{a}\right)+Q_{r}+Q_{\text {cond }}
$$

With Eq. (6), it is possible to transform Eq. (1) into:

$$
\begin{aligned}
& \frac{P-Q_{r}-Q_{\text {cond }}}{\left(T_{w}-T_{a}\right)^{5 / 4}}= \\
& 0.508 \cdot \lambda \cdot 2 A_{w} \cdot \beta^{1 / 4} \cdot v^{-1 / 2} \cdot x^{-1 / 4}\left(\frac{\operatorname{Pr}^{2}}{0.952+\operatorname{Pr}}\right)^{1 / 4} w^{1 / 4}
\end{aligned}
$$

The difference of the dissipated power and the radiated heat and conducted heat normalised by the temperature difference of resistor and ambient to the power 1.25 is proportional to $\mathrm{w}^{1 / 4}$ In a graphical representation, this equation is a line when $w^{1 / 4}$ is the abscissa and $\frac{P-Q_{r}-Q_{\text {cond }}}{\left(T w-T_{a}\right)^{5 / 4}}$ the ordinate. The theoretical slope of this line is:

$\frac{1}{l} \int_{0}^{l} 0.508 \lambda \cdot 2 A_{w} \cdot \beta^{1 / 4} \cdot v^{-1 / 2} \cdot x^{-1 / 4}\left(\frac{\operatorname{Pr}^{2}}{0.952+\operatorname{Pr}}\right)^{1 / 4} d x$ 
This slope is determined by the fluid properties (i.e. air) at the average temperature and by the characteristics of the flat resistor (surface area $A_{w}$ and height I). This theoretical slope will be used to validate the exactness of the experiment.

\section{Experimental setup}

A flat electrical ceramic resistor of $25.5 \mathrm{~mm} \times 25.5 \mathrm{~mm}$ is used as heat source. A flat resistor was chosen to be able to use the theory of a laminar flow on a constant-temperature, vertical, flat plate as validation of the experiment. The resistor has to have a homogeneous temperature on its surface in order to do accurate measurements, therefore a ceramics resistor was chosen. The small size of the resistor makes it possible to use the centrifuge for the experiment. The resistor is placed inside a hermetically sealed Plexiglas cylinder with a radius of $70 \mathrm{~mm}$ and a height of $300 \mathrm{~mm}$. The cylinder hangs from an arm of a centrifuge (Fig.1). By rotating the centrifuge, the acceleration inside the cylinder becomes greater than $\mathrm{g}$. The cylinder was perfectly sealed to prevent the natural convection cooling of the heat source being disturbed by external airflows during rotation. The power source for the resistor also has to be inside the cylinder for practical reasons. It was difficult to make a connection between an external power source and the resistor when the centrifuge rotates. Therefore six rechargeable AA-batteries of $1.2 \mathrm{~V}$ each were chosen as power source. The voltage over the resistor was kept constant at $5 \mathrm{~V}$ with a voltage stabilisator. The resistor dissipates $1.63 \mathrm{~W}$ and has a mean temperature of $82^{\circ} \mathrm{C}$ at $1 \mathrm{~g}$. An insulating layer of $20 \mathrm{~mm}$ thickness was put between resistor and battery. This way, heat coming from the battery does not disturb the resistor cooling and conductive heat transfer from resistor to battery is avoided. The heat source is cooled by natural convection, radiation and a small amount of conduction.

The centrifugal acceleration of an object hanging beneath the centrifuge depends on the radius of the centrifuge and the rotational speed. The total acceleration is also influenced by gravitation. All the forces and accelerations are shown in Fig. 1 [9]. When the rotational speed increases, the cylinder will be swung more to the outside. The radius at which the resistor rotates is bigger than $R$ (Fig. 1):

$$
R_{1}=R+\Delta R
$$

with

$$
\Delta R=\sin \alpha \cdot H_{1}
$$

The acceleration is given by the following expression:

$$
w=\sqrt{\left[\frac{\left(\frac{2 \pi R_{1} f}{60}\right)^{2}}{R_{1}}\right]^{2}+g^{2}}
$$

This acceleration lies along the axis of the cylinder, because the connection between the centrifuge and the cylinder is made with a hinged joint. The acceleration is controlled by changing the rotational speed of the centrifuge. The centrifuge is driven by a motor, which can be set to a specific speed by a controller.
The dissipated power, the temperature of the resistor, and the ambient temperature in the cylinder were measured. The dissipated power is the same for all measurements point due to the voltage limiter. The temperature of the resistor was measured with a thermographic camera. There is no contact needed with the resistor, so conduction is avoided and the environment is not disturbed. The cylinder is made out of Plexiglas, which is not transparent to IR-radiation. Therefore the cylinder is equipped with a window of $\mathrm{CaF}_{2}$ (calcium fluoride). $\mathrm{CaF}_{2}$ has a high transmittance for IR-radiation. The resistor was placed right behind this window. First the camera was focused on the surface of the resistor. Therefore the container was hung at the angle $\alpha$ (Fig. 1), corresponding the angle of the desired rotational speed, under the non-rotating centrifuge to be able to focus correctly. This gives a static image as shown in Fig. 2.

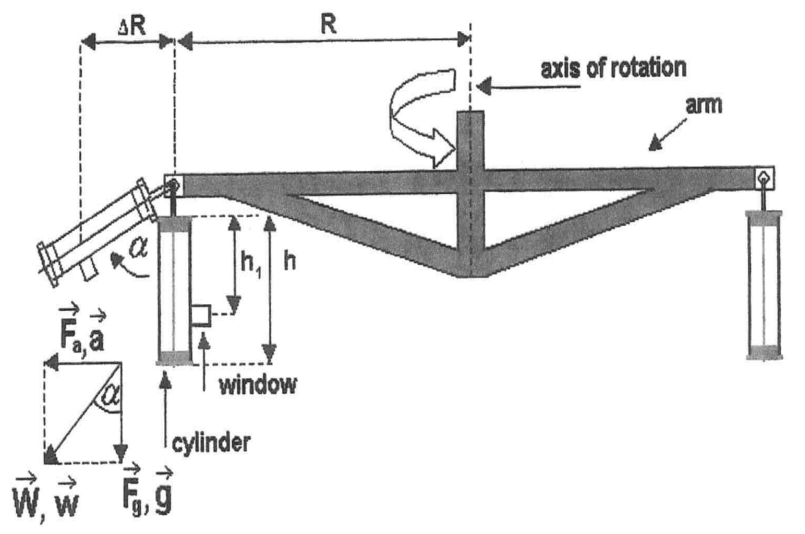

Fig. 1. Scheme of experimental setup

The centrifuge is activated at the desired rotational speed. After a few minutes steady-state condition is obtained. The infrared camera is connected to a computer on which the images are displayed. The picture is frozen when the cylinder passes the camera. Now the cylinder is inclined in the frame of the image (Fig.3 and Fig.4). The computer scans the images of the camera from top to bottom. So the second line of the image is shifted compared to the first line, the third shifted compared to the second, and so on. The faster the centrifuge rotates, the faster the cylinder passes the camera, and the cylinder will

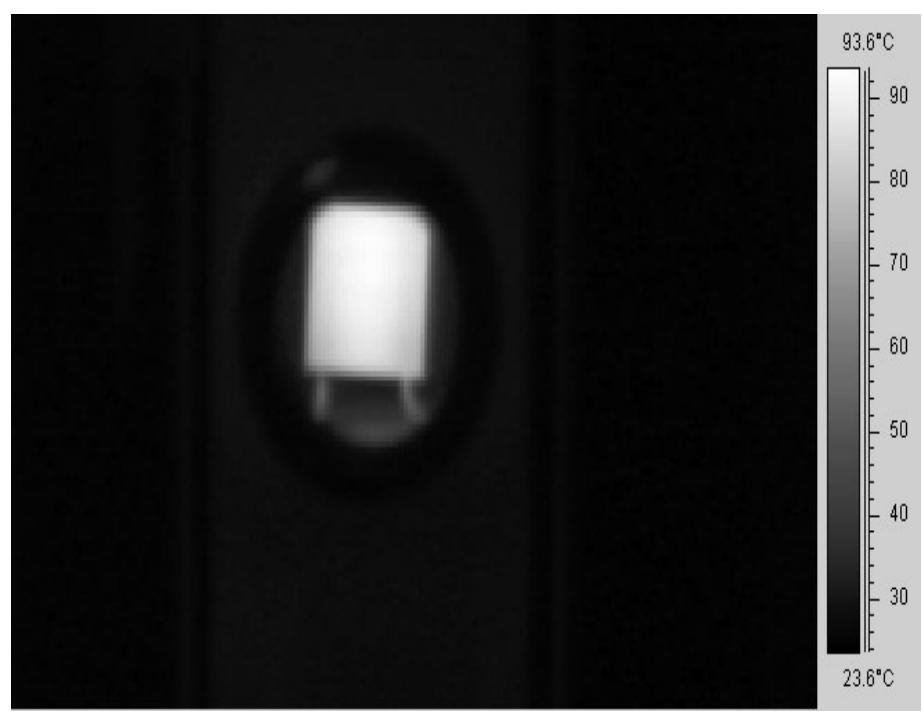

Fig. 2. Cylinder with resistor for non-rotating centrifuge 


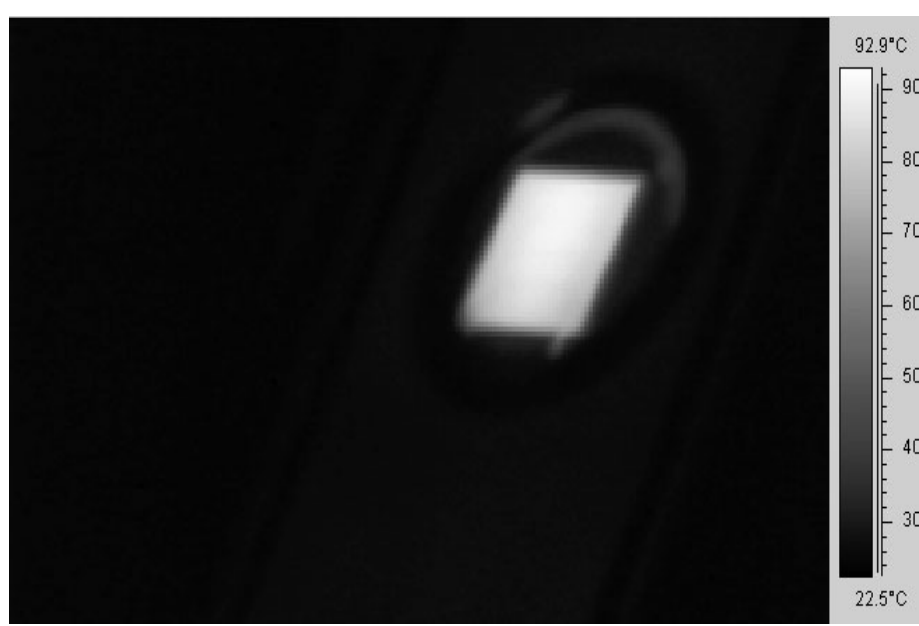

Fig. 3. Image at $12 \mathrm{t} / \mathrm{min}$ of centrifuge

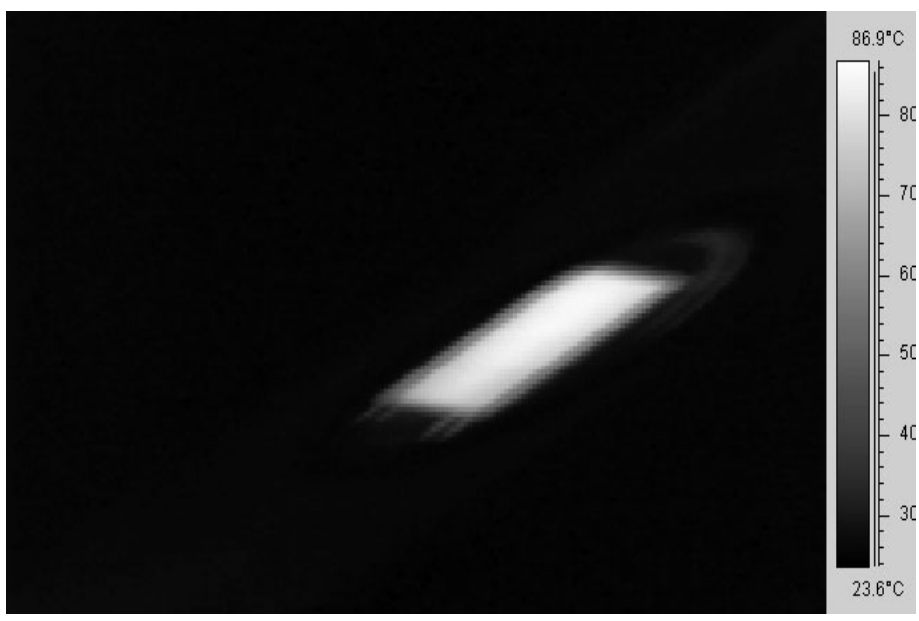

Fig. 4. Image at $36 \mathrm{t} / \mathrm{min}$ of centrifuge

have more inclination. In Fig. 3 and Fig. 4 two images are shown at different speeds of the centrifuge. Afterwards, the images are processed, i.e. the mean temperature of the resistor surface is determined with the computer. This is difficult to execute precisely and can induce some extra errors.

The ambient temperature in the cylinder cannot be measured with the thermographic camera because the rest of the cylinder was made out of Plexiglas. Also, a thermographic camera can only measure surface temperatures and no air temperatures at a specific point. A thermocouple was used to measure the ambient temperature. This thermocouple was equipped with a programmable recorder. It took temperature samples every minute and saved them inside the recorder. The recorder was attached to the outside of the cylinder. The thermocouple was placed inside the cylinder and connected to the recorder with a small wire.

It is important to mention the influence of the Coriolis force. If the cylinder is rotated with a centrifuge, not only a centrifugal force will work on the cylinder. Because of the air movement inside the cylinder, also a relative force (Coriolis force) works on the air. This force works sideways on the air in the cylinder. It is possible that this can influence the natural convection. The Coriolis force is:

$$
\begin{aligned}
& F_{c}=2 m \cdot\left(\omega \times v_{l}\right) \\
& F_{c}=2 m \cdot \omega \cdot v_{l} \cdot \sin \alpha
\end{aligned}
$$

The air speed $v_{l}$ in the cylinder is estimated to be $1 \mathrm{~cm} / \mathrm{s}$. The Coriolis force is calculated for each measurement point and the biggest influence is at $w=53$ and is only $0.2 \%$ of the centrifugal force, so it can be neglected. So natural convection is almost completely induced by the centrifugal force.

\section{Results}

The experiment has been executed two times. Two series of data points are obtained, which can be plotted in a graph with $\mathrm{w}^{1 / 4}$ at the $\mathrm{x}$-axis and $\frac{P}{\left(T_{w}^{4}-T_{a}^{4}\right)^{5 / 4}}$ at the $\mathrm{y}$-axis. According to the physics, a linear regression line, which represents the total heat transfer (natural convection + radiation + conduction), can be drawn between the data points of each measurement. Theoretically, the two regression lines should coincide because the same material has been used for both experiments. In reality, they will not coincide due to measurement errors and varying room temperature. The slope of the linear regression line of the first measurement is smaller than that of the second one (Fig. 5). This difference is due to measurement errors.

A regression line that represents the amount of radiation can also be drawn. Only the resistor temperature $T_{w}$ and the ambient temperature $T_{a}$ are needed. The data points with $\frac{2 \sigma A_{w}\left(T_{w}^{4}-T_{a}^{4}\right)}{\left(T_{w}-T_{a}\right)^{5 / 4}}$ as ordinates and the same abscissa are put on the graph. The two lines for radiation coincide almost perfectly. Also a regression line for the amount of conduction

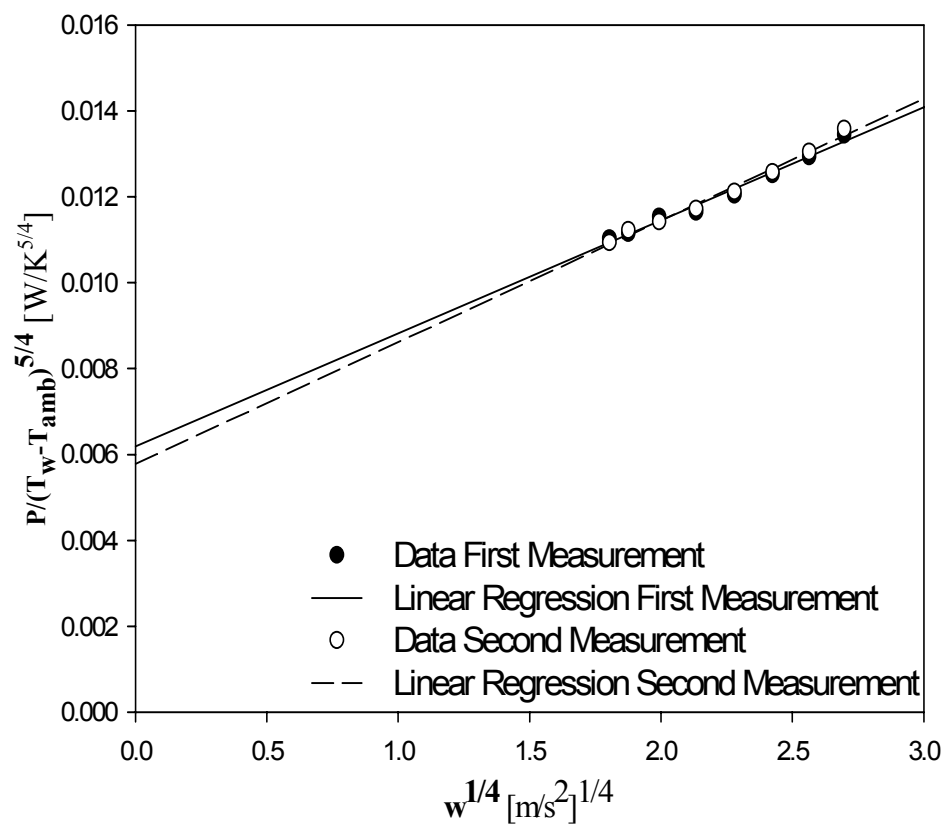

Fig. 5. Linear regression line for total heat transfer for the two measurements 
through the wires that connect the resistor with the batteries, can be drawn. Therefore $\frac{2 \cdot A_{\text {wire }} \cdot \lambda_{\text {wire }} \cdot\left(T_{w}-T_{e}\right)}{l_{\text {wire }}\left(T_{w}-T_{a}\right)^{5 / 4}}$ is used as ordinates. In Fig. 6, the lines for radiation, conduction and the total heat transfer are drawn for the data of the two measurements put together. The line for radiation has a small slope and shows that the amount of radiation does not remain constant with changing $w$. The difference between the regression line for total heat transfer and the regression lines for radiation and conduction represents the natural convection. A graph for natural convection can also be drawn. The abscissas remain the same, but ordinates are now $\frac{P-Q_{r}-Q_{\text {cond }}}{\left(T_{w}-T_{a}\right)^{5 / 4}}$. The data points in this graph represent the natural convection and again a regression line can be drawn for each measurement. In Fig. 7 the two regression lines for natural convection are drawn. The exactness of the experiment still has to be validated to the governing equations.

\section{Error Analysis}

To validate the experiment, the experimental slope has to be compared with the theoretical one. The theoretical slope was calculated for natural convection only. Therefore the graph for natural convection was used. First an error estimation was done on the regression lines for natural convection. The least-square method is used for error calculation. The error for the first regression line is $10.3 \%$ for the slope. The absolute error for the intercept is $6.037 \cdot 10^{-4}$. The intercept is $1.567 \cdot 10^{-3}$. For the second regression line the error is $9.6 \%$ for the slope and the absolute error for the intercept is $6.062 \cdot 10^{-4}$ for an intercept of $1.17 \cdot 10^{-3}$. This is $50 \%$ of the intercept. In Fig. 6

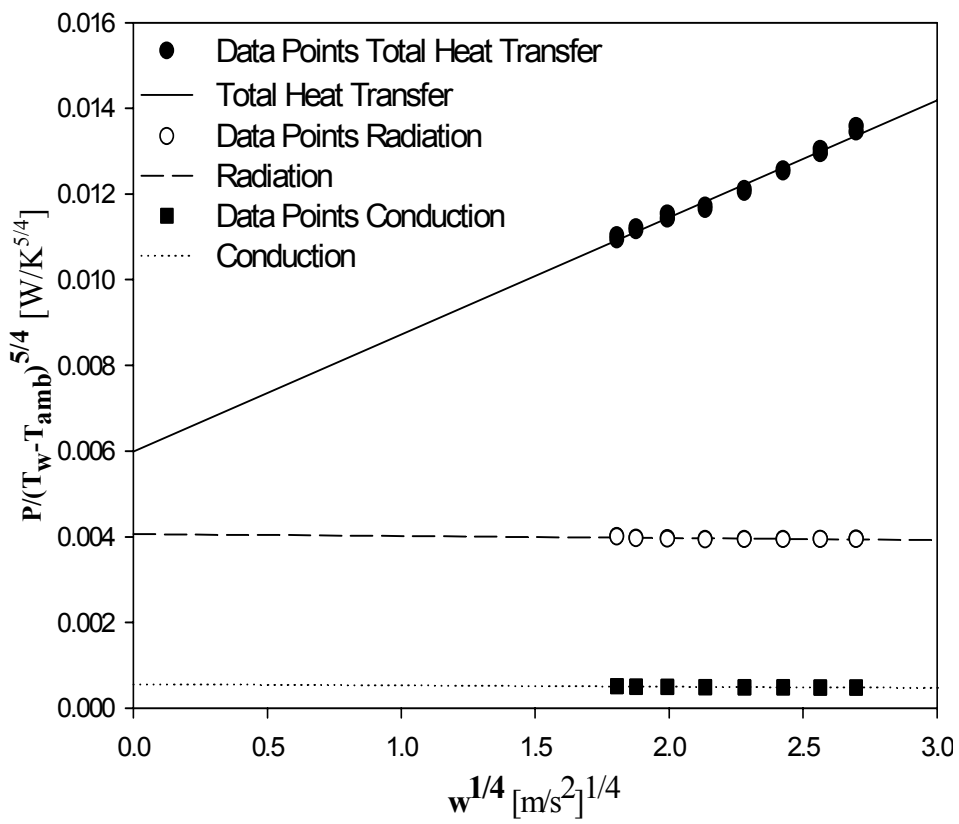

Fig. 6. Linear regression for total heat transfer, radiation and conduction for the two measurements together the regression line of the two measurements added together is used. This regression line is based on more data points and has the smallest error at the intercept. The errors are $7 \%$ for the slope and $4.277 \cdot 10^{-4}$ for an intercept of $1.369 \cdot 10^{-3}$.

There is also an error for the measurements: the temperature measurements, the voltage measurements and the surface determination of the resistor. These errors were accounted for in the error determination of the slope and intercept of the linear regression lines. The absolute error is $0.9^{\circ} \mathrm{C}$ for the resistor temperature due to the accuracy of the thermographic camera. The thermocouple is more accurate than the camera, and the error is $0.5^{\circ} \mathrm{C}$. The error made on the voltage determination is determined at $0.01 \mathrm{~V}$ and this is small compared with the measurement values that are all $4.98 \mathrm{~V}$. Finally the error for the length and height measurement is set to $0.000025 \mathrm{~m}$ (measurement accuracy). With these values, the error on the $y$-axis values for the graph of natural convection (Fig. 8) can be determined. It is determined by the error on the total heat transfer, radiation, conduction and $\left(T_{w}-T_{a}\right)^{5 / 4}$. The error on the total heat transfer is determined by the error on the voltage measurement, being $0.4 \%$. The error for the radiation depends on the error of the temperature measurements and the dimensions of the resistor (Eq. 4) and is determined at $2.6 \%$. The error for conduction is $13.7 \%$. The resulting error for natural convection is $1.6 \%$. The error for the $y$-axis values is $3.2 \%$. The values at the $x$-axis are assumed to be without error. The value of $w^{1 / 4}$ is determined by Eq. (11) and depends on the accuracy of the rotational velocity of the centrifuge, thus on the correctness of the rotational speed of the motor. It is assumed that this error is very small. Even if this error is 0.5 rotations per minute, the relative error on the acceleration $w^{1 / 4}$ is of magnitude $0.014 \%$. Furthermore, the two different measurements are almost all done at the same speeds, so the error on the x-axis between the two measurements is the same for both. The error on the abscissa

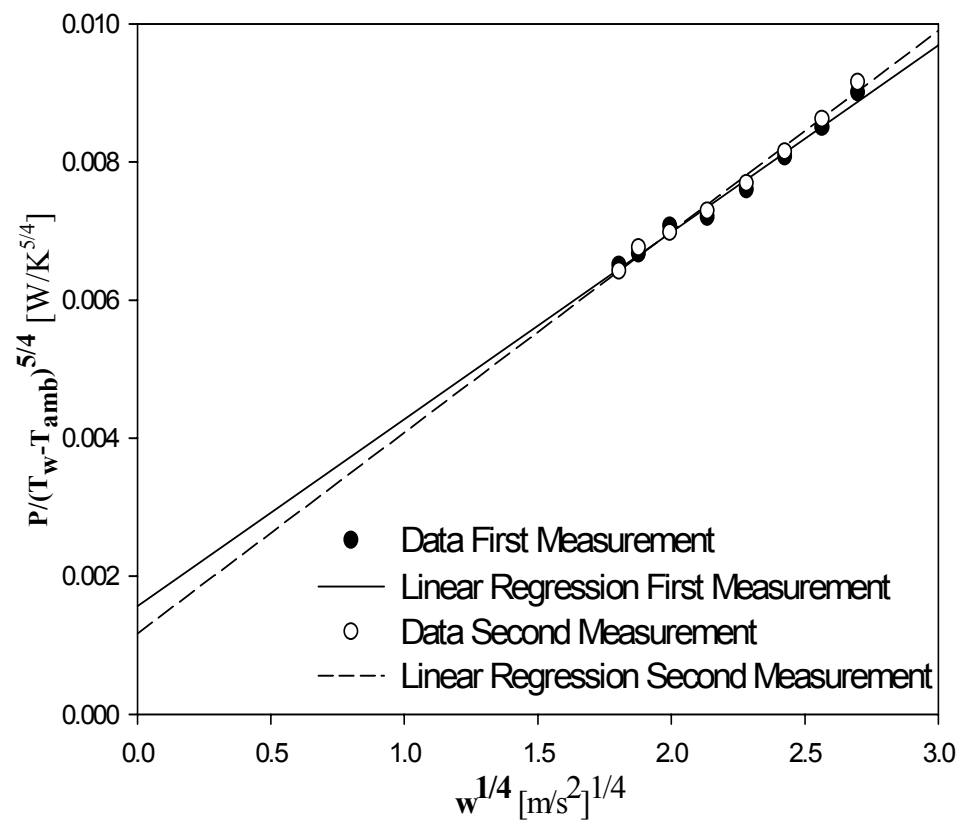

Fig. 7. Linear regression line for natural convection for the two measurements 
can be neglected because of these two reasons.

The reliability of the experiment is also checked. Because the cylinder rotates at high speeds and is not insulated, there will be convective heat transfer from the cylinder to the environment. In the cylinder $1.63 \mathrm{~W}$ of heat is produced. The percentage of heat transferred to the environment has to be checked. To have reliable experiments, this amount has to be almost the same for the highest as for the lowest speed. Calculations show us that at the highest speed at $66 \mathrm{t} / \mathrm{min}$, $0.212 \mathrm{~W}$ is transferred to the environment. This is $13 \%$ of the total produced heat. At $18 \mathrm{t} / \mathrm{min}$ (lowest speed), it is $0.262 \mathrm{~W}$ or $16.1 \%$. This difference is small enough to accept that the experiment is reliable. For the heat transfer from the inside of the cylinder to the environment, the most important factor is the convection coefficient inside the cylinder (natural convection). This explains why the heat transferred to the environment does not change much with changing rotational speed. The convection coefficient outside the cylinder is much higher (10 times). This causes the wall temperature of the cylinder to be as high as the ambient temperature and so the amount of radiation to the environment from the cylinder is the same for all speeds.

\section{Evaluation Of The Results}

The intercept of the regression line for the total heat transfer (Fig. 6) determines the amount of radiation at $w=0$ and a small amount of conduction through the connecting wires and the stagnant air in the cylinder. This is obtained by extrapolating the line to zero gravity. At $w=0$, the driving force for natural convection has completely disappeared, thus there is no natural convection anymore and an estimate for the amount of radiation can be made. This amount decreases with increasing $w$ due to a lower resistor temperature $\mathrm{T}_{\mathrm{w}}$, and if it is normalised to $\left(T_{w}-T_{a}\right)^{5 / 4}$, it decreases very slightly (Fig. 6). In Fig. 7, the regression lines for natural convection should have an intercept that lies a bit above zero due to conduction through the air. With the experimental results, intercepts of $1.567 \cdot 10^{-3}$ and $1.17 \cdot 10^{-3}$ were found. These intercepts seem too big but the error for the intercepts is almost the half of the intercept as mentioned above. Also, the amount of conduction is underestimated a bit, because the temperature at the end of the connecting wires is measured for a non-rotating cylinder. These wires end in the lower part of the cylinder that contains the batteries and the voltage stabilisator. It is isolated from the upper part that contains the resistor. When the cylinder is rotating, the lower section of the cylinder will be cooled better by forced convection on the outside of the cylinder, so the temperature in this part will be lower, thus $T_{e}$ will be lower. The amount of conduction (Eq. 5) will be bigger so the intercept will drop and approach to zero.

The experimental slope can be compared with the theoretical one. In the theory, the fluid characteristics are used for air at a temperature of $50^{\circ} \mathrm{C}$ (average between $25^{\circ} \mathrm{C}$ and $75^{\circ} \mathrm{C}$ ). The slope is then 0.002648 . The experimental slope is $0.002706 \pm 0.00002777$ for the first measurement and 0.00291 \pm 0.0002795 for the second one. The theoretical value lies in the error margin of the experimental slope for both measurements. In the experiment $59 \%$ of heat is transferred by natural convection at $w=g\left(w^{1 / 4}=1.77\right)$. At $6 g\left(w^{1 / 4}=2.77\right)$ it has risen to $67.5 \%$. There is clearly an improvement of the natural convection and of the global cooling of the resistor. The surface temperature of the resistor drops from $81^{\circ} \mathrm{C}$ at $1.25 \mathrm{~g}$ to $71^{\circ} \mathrm{C}$ at $6 \mathrm{~g}$.

\section{Conclusion}

Radiation and natural convection can easily be determined from the experimental results without any difficult calculations. It is a good method to separate natural convection from radiation and conduction with acceptable exactness, because there is a good similarity between theory and experiment. Moreover, the results of first and second experiment show that the technique has a good reproduciblity.

\section{REFERENCES}

[1] Kim K. H., Hyun J. M., Kwak H. S., 2001, "Buoyant convection in a side-heated cavity under gravity and oscillations", International Journal of Heat and Mass Transfer, 44, pp.857-861.

[2] Jin, Y. Y. and Chen, C. F., 1996, "Effect of gravity modulation on natural convection in a vertical slot", International Journal of Heat and Mass Transfer, 40, pp. 1411-1426.

[3] Ben Q. Li, 1996, "g-Jitter induced free convection in a transverse magnetic field", International Journal of Heat and Mass Transfer, 39, pp.2853-2860.

[4] Wenxian Lin, Armfield S. W., 1999, "Direct simulation of natural convection cooling in a vertical circular cylinder", International Journal of Heat and Mass Transfer, 42, pp.4117-4130.

[5] Ekundayo C.O., Probert S.D., M. Newborough, 1998, "Heat transfers from a horizontal cylinder in a rectangular enclosure", Applied Energy, 61, pp.57-78.

[6] Greger R. and Rath H.J., 1995, "Measurements of the thermal conductivity of fluids with low viscosity under reduced gravity conditions using the transient hot-wire technique", International Journal of Heat and Mass Transfer, 39, pp.1105-1110.

[7] Willockx A. et al, 2004, Seperation of natural convection and radiation by changing rotational acceleration, ASME Heat Transfer/Fluids Engineering Summer Conference.

[8] Kays W. M. and Crawford M. E., 1993, Convective Heat and Mass Transfer, McGraw-Hill, Inc., Singapore.

[9] De Cramer M. and Stouf A., 2003, Invloed van de gravitatieversnelling op de koeling van elektronische componenten, Thesis, Hogeschool Gent, Gent, Belgium. 